\title{
Visceral adiposity index is a better predictor of unhealthy metabolic phenotype than traditional adiposity measures: results from a population-based study
}

\author{
Fabrícia Geralda Ferreira ${ }^{1, *}$, Leidjaira Lopes Juvanhol ${ }^{2}$, \\ Danielle Cristina Guimarães da Silva ${ }^{3}$ and Giana Zarbato Longo ${ }^{2}$ \\ ${ }^{1}$ Escola Preparatória de Cadetes do AR (EPCAR), Street Santos Dumont 149, Barbacena - MG, 36205-058, Brazil: \\ ${ }^{2}$ Department of Nutrition and Health, Universidade Federal de Viçosa, Viçosa, MG, Brazil: ${ }^{3}$ Universidade Federal \\ do Oeste da Bahia (UFOB), Barreiras, BA, Brazil
}

Submitted 5 February 2018: Final revision received 11 October 2018: Accepted 29 0ctober 2018: First published online 6 December 2018

\begin{abstract}
Objective: The present study aimed to investigate whether the visceral adiposity index (VAI) is an effective predictor to identify unhealthy metabolic phenotype by comparing normal-weight and overweight individuals.

Design: A population-based cross-sectional study. Data were collected by interviews, anthropometric evaluation, dietetic, clinical and laboratory tests. The area under the receiver-operating characteristic curve (AUC) and prevalence ratio (PR), obtained from Poisson regression, were used to compare the predictive capacity of the obesity indicators evaluated (VAI, BMI, waist and neck circumference, waist-to-height and waist-to-hip ratios) and their association with the unhealthy metabolic phenotype. All analyses were stratified by sex and by nutritional status.

Setting: Viçosa, Minas Gerais, Brazil.

Participants: A total of 854 Brazilian adults (20-59 years old) of both sexes.

Results: VAI was the best predictor for unhealthy metabolic phenotype among men (AUC $=0.865)$ and women (AUC $=0.843)$ at normal weight. VAI also had the best predictive capacity among overweight women (AUC=0.903). Among overweight men, its accuracy (AUC $=0.830$ ) was higher than that of waist-to-hip ratio. In the adjusted regression models, VAI was the indicator most strongly associated with the unhealthy metabolic phenotype, especially among those with normal weight $(\mathrm{PR}=6 \cdot 74 ; 95 \%$ CI 3.15, 14.42 for men; $\mathrm{PR}=7 \cdot 14 ; 95 \%$ CI $3 \cdot 79$, 13.44 for women).

Conclusions: VAI has better predictive capacity in detecting unhealthy metabolic phenotype than conventional anthropometric indicators, regardless of nutritional status and sex.
\end{abstract}

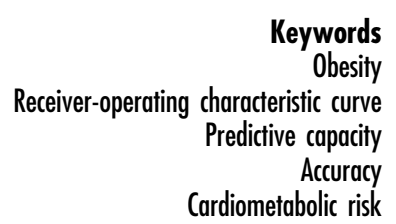

Obesity is considered a multifactorial condition $^{(1)}$ that predisposes the individual to the development of several diseases, including CVD, type 2 diabetes and some types of cancer $^{(1,2)}$. However, it has been found that not all obese individuals develop these disorders, as well as that some normal-weight individuals have an unfavourable cardiometabolic profile $^{(3)}$. Thus, there are probably other determinants of obesity-related complications, in addition to overweight ${ }^{(4)}$.

BMI is the most commonly used diagnostic measure in the identification of obesity in population studies and clinical practice ${ }^{(5)}$. However, as previously stated, a portion of individuals who are in the BMI range considered adequate have metabolic disorders characteristic of obese individuals ${ }^{(3)}$. These individuals, according to the literature, have the phenotype called 'metabolically unhealthy normal weight ${ }^{(6,7)}$, with early signs of insulin resistance, dyslipidaemia and hypertension. The main implication related to this group is that since individuals are classified as normal weight and thus apparently healthy, metabolic changes are detected late and therefore are not properly treated.

On the other hand, overweight/obese individuals showing no metabolic changes ${ }^{(3)}$ are classified as 'metabolically healthy overweight' phenotype ${ }^{(7)}$. Nevertheless, recent evidence indicates that this condition may be 
transient, since follow-ups over a longer period have shown the progression to a metabolically unhealthy phenotype ${ }^{(8-11)}$. In addition, 'metabolically healthy overweight' is not a totally benign condition since it has been demonstrated that individuals with this phenotype are at a higher risk of developing type 2 diabetes and CVD in relation to normal-weight and metabolically healthy individuals $^{(12)}$.

Although the identification of individuals belonging to each of the phenotypes is essential for the most appropriate therapy to be adopted ${ }^{(13)}$, the application of the same is hampered by the absence of an internationally standardized definition $^{(14)}$. The most commonly adopted definitions in the literature use a set of unfavourable metabolic condition markers, such TAG, HDL-cholesterol (HDL-C), total cholesterol, glycaemia, homeostatic model assessment of insulin resistance (HOMA-IR), blood pressure, waist circumference (WC) and ultra-sensitive $\mathrm{C}$-reactive protein (us-CRP), which may hamper its wider use in clinical practice. Thus, finding an index to accurately identify the unhealthy metabolic phenotype with fewer markers is crucial. Another important aspect is that these different definitions take no account of specific markers of visceral adipose tissue accumulation, which has been pointed out as one of the main causes of the metabolic deterioration of individuals ${ }^{(8,15)}$. Considering that the direct measurement of this tissue requires the use of expensive imaging techniques that are not feasible in the context of public health, alternative measures that estimate visceral adiposity must be investigated.

In this context, the visceral adiposity index (VAI) has been described as a useful indicator of the visceral fat function associated with cardiometabolic risk ${ }^{(16)}$, since it requires no expensive technique for its calculation. VAI is a sex-specific index based on anthropometric (BMI and WC) and lipid (TAG and HDL-C levels) parameters, which was developed from an adult population without associated diseases $^{(16)}$. It was validated as a simple replacement marker of visceral adiposity and adipose dysfunction obtained by MRI ${ }^{(16)}$. Therefore, its use in the identification of the unhealthy metabolic phenotype may represent an interesting alternative.

However, to the best of our knowledge, no study has so far evaluated the predictive capacity of VAI for identifying the unhealthy metabolic phenotype comparing normalweight and overweight individuals. In addition, no population-based studies have been found that surveyed the prevalence of different obesity phenotypes in the Brazilian population.

Thus, the present study aimed to verify whether VAI is an effective predictor in identifying unhealthy metabolic phenotype between normal-weight and overweight individuals and compared it with traditional indicators of obesity in a representative sample of Brazilian adults.

\section{Methodology}

\section{Study design and participants}

The present study is a cross-sectional, population-based study on health conditions of a representative sample of the adult population in Viçosa, Minas Gerais, Brazil, conducted from 2012 to 2014. Details of the study procedures are described in Segheto et al. ${ }^{(17)}$.

The study included adult individuals aged 20-59 years of both sexes and residing in the urban area of the city. For the sample calculation, we adopted the following parameters: estimated population of 43431 individuals, confidence level of $95 \%$, expected prevalence of $50 \%$ (multiple outcomes) $)^{(18)}$ and sample error of $4.5 \%$. In addition, a design effect of 1.6 and adding $10 \%$ for losses and refusals and $10 \%$ to control confounding factors were applied. The sample size was estimated as 901 individuals.

Probabilistic sampling was used without replacement, by double-stage sampling (census and domicile), and 1229 household interviews were carried out. Of the total number of interviewees, 331 individuals did not complete the laboratory tests and forty-four had serum TAG $\geq 279 \mathrm{mg} / \mathrm{dl}$ and/or $\mathrm{BMI} \geq 40 \cdot 0 \mathrm{~kg} / \mathrm{m}^{2}$, who were excluded from the study as recommended by the literature for VAI calculation $^{(19)}$. Thus, the final sample of the present study was composed of 854 individuals (Fig. 1). No significant statistical difference was found for sociodemographic characteristics between the original sample and the one used in the study (data not shown).

\section{Measurements}

\section{Biochemical parameters}

Blood samples were collected by venepuncture using the Vacutainer system (Becton Dickinson, Plymouth, UK) after

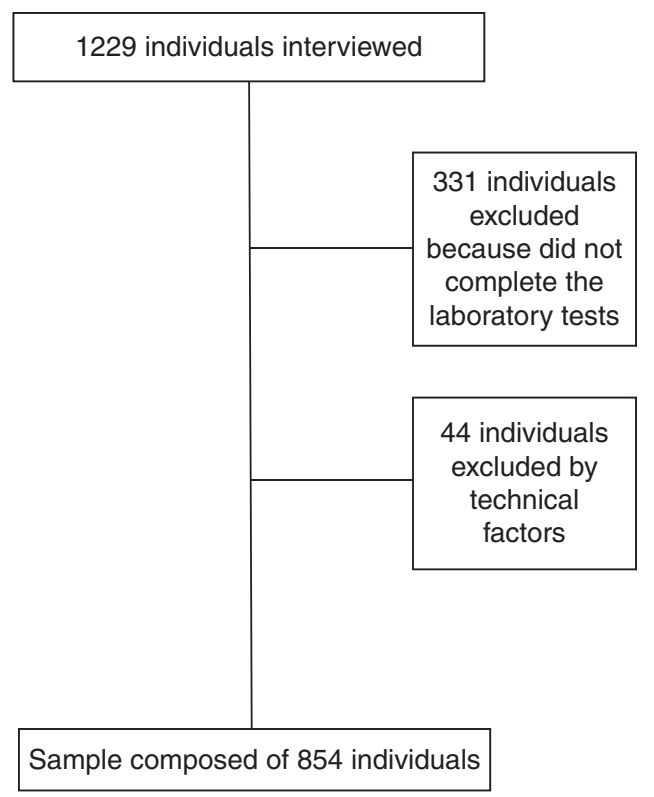

Fig. 1 Flowchart of participant selection 
$12 \mathrm{~h}$ of fasting. Fasting glucose was determined by the enzymatic glucose-oxidase method. TAG and HDL-C concentrations were measured by the enzymatic colorimetric method. Plasma insulin was determined by ELISA, using the Human Insulin ELISA Kit (Linco Research ${ }^{\circledR}$, St. Charles, MO, USA). Insulin resistance was estimated by the homeostatic model assessment: HOMA-IR $=[$ fasting insulin $(\mu \mathrm{U} / \mathrm{ml}) \times$ fasting glucose $(\mathrm{mmol} / \mathrm{l})] / 22 \cdot 5^{(20)}$. us-CRP was determined by the immunoturbidimetric assay (Bioclin ${ }^{\circledR}$, Quimbasa Quimica Básica, Belo Horizonte, MG, Brazil).

\section{Anthropometric parameters and body composition}

Weight was measured with an electronic digital scale (TANITA $^{\circledR}$ model Ironman BC-554, Tokyo, Japan), certified by Inmetro, with a capacity of $150 \mathrm{~kg}$ and a precision of $100 \mathrm{~g}$. Height was measured using a fixed stem stadiometer, coupled to the wall without a footboard (WELMY ${ }^{\circledR}$, Santa Bárbara D'Oeste, SP, Brazil), with $2.5 \mathrm{~m}$ extension and $0.1 \mathrm{~cm}$ resolution. BMI was calculated as weight (in kilograms) divided by the square of the height (in metres) ${ }^{(21)}$. WC was measured in centimetres with an inelastic measuring tape (Sanny ${ }^{\circledR}$, São Paulo, SP, Brazil) at the midpoint between the last rib and the iliac crest, at the end of a normal exhalation. Waist-to-hip ratio (WHR) was calculated as the ratio between WC and hip circumference measured at the maximal gluteal protuberance ${ }^{(21)}$. Waist-to-height ratio (WHtR) was calculated as the ratio between WC and height ${ }^{(22)}$. Neck circumference (NC) was measured in centimetres, just below the laryngeal prominence, with individuals standing and their head positioned in the Frankfurt plane ${ }^{(23)}$, using a flexible and inelastic metric tape (Sanny ${ }^{\circledR}$, São Paulo, SP, Brazil). All anthropometric measurements were performed in triplicate by one experienced examiner, considering the average values. VAI was calculated using sex-specific formulas ${ }^{(16)}$ : VAI, males $=[\mathrm{WC} / 39.68+(1.88 \times \mathrm{BMI})] \times(\mathrm{TAG} / 1.03) \times$ $(1 \cdot 31 / \mathrm{HDL}-\mathrm{C})$ and VAI, females $=[\mathrm{WC} / 36.58+(1.89 \times$ $\mathrm{BMI})] \times(\mathrm{TAG} / 0 \cdot 81) \times(1 \cdot 52 / \mathrm{HDL}-\mathrm{C})$, with TAG and HDL-C levels expressed as $\mathrm{mmol} / \mathrm{l}$.

Female body composition was estimated by the triceps, abdomen and suprailiac skinfolds, and male body composition by triceps, pectoral and subscapular skinfolds. Skinfold data were used in sex-specific equations to calculate body density ${ }^{(24,25)}$, then the fat percentage was estimated by the Siri equation ${ }^{(26)}$. The skinfolds were measured using a Lange $^{\circledR}$ calliper (Beta Technology Incorporated, Cambridge, MA, USA) with $1 \mathrm{~mm}$ precision.

\section{Clinical parameters}

Systolic blood pressure (SBP) and diastolic blood pressure (DBP) were measured in duplicate using an automatic insufflation blood pressure monitor $\left(\mathrm{OMRON}^{\circledR}\right.$ model HEM-741 CINT, Tokyo, Japan), calibrated and certified by Inmetro. The first measure was obtained after $5 \mathrm{~min}$ rest and the second $15 \mathrm{~min}$ after the first measurement. The mean of the two measurements was considered for analysis.
Leisure-time physical activity and energy intake

The leisure-time physical activity (LTPA) was evaluated by the International Physical Activity Questionnaire (IPAQ), long form, using the fourth domain. LTPA was calculated by adding the time spent in moderate physical and walking activities plus twice the time spent in vigorous activities ${ }^{(27)}$.

The participants' energy intake was estimated using an FFQ previously validated for the population ${ }^{(28)}$.

\section{Definition of phenotypes}

The sample was categorized into four phenotypes: (i) metabolically healthy normal weight (MHNW); (ii) metabolically unhealthy normal weight (MUHNW); (iii) metabolically healthy overweight (MHO); and (iv) metabolically unhealthy overweight (MUHO).

Phenotype definition was based on Wildman et al.'s criteria $^{(7)}$, wherein individuals are considered metabolically unhealthy when two or more of the following cardiometabolic abnormalities are present: SBP/DBP $\geq 130 / 85 \mathrm{mmHg}$ or taking antihypertensive drugs; TAG $\geq 150 \mathrm{mg} / \mathrm{dl}$; HDL$\mathrm{C}<40 \mathrm{mg} / \mathrm{dl}$ in males and $<50 \mathrm{mg} / \mathrm{dl}$ in females or use of lipid-lowering medication; glucose $\geq 100 \mathrm{mg} / \mathrm{dl}$ or use of hypoglyacemic medication; and HOMA-IR and usCRP $>90$ th percentile. Individuals with $\mathrm{BMI} \geq 25.0 \mathrm{~kg} / \mathrm{m}^{2}$ were considered overweight and those below this value were considered normal weight ${ }^{(21)}$.

\section{Statistical analysis}

All variables were examined for normality by the ShapiroWilk test and graphical assessment. The non-normally distributed variables are presented as medians and interquartile ranges. The Kruskal-Wallis test was used to compare groups with Dunn's post hoc test. Receiver-operating characteristic (ROC) curves were constructed to compare the predictive capacity of VAI with other indicators of adiposity (WC, WHtR, WHR and NC), aiming to detect unhealthy metabolic phenotype among normal-weight and overweight individuals. The overall accuracy was calculated using the area under the ROC curve (AUC) and AUC $\leq 0.5$ was indicative of lack of discriminatory ability ${ }^{(29)}$. Based on the literature ${ }^{(29,30)}$, $\mathrm{AUC} \geq 0.90$ is considered to indicate excellent performance, AUC between 0.80 and 0.90 to indicate good performance, AUC between 0.70 and 0.80 to indicate fair performance and AUC between 0.50 and 0.70 to indicate poor performance. The ROC curves were also used to determine the optimal cut-off points for the different adiposity indicators, and those with the best balance between the sensitivity and specificity values were selected. From the cut-off points obtained, the association of the adiposity indicators with the unhealthy metabolic phenotype in the two analysed groups (normal weight and excess weight) was examined by Poisson regression analysis with robust variance. The models were adjusted for age (continuous, in years), schooling (categorized as $0-4,5-8,9-11$ and $\geq 12$ years of education) and socio-economic status (categorized as high, intermediate and 
low). Prevalence ratios (PR) and their respective 95\% CI were calculated. The statistical significance was $\alpha=0 \cdot 05$. All statistical analyses were performed using the statistical software package Stata version 13.0.

\section{Results}

Of the 854 individuals evaluated, the prevalence in the sample for each of the phenotypes was 51.05 (95\% CI 47.69, 54.40) \% for MHNW, 10.07 (95\% CI 8.22, 12.28) \% for MUHNW, 21.78 (95\% CI 19.13, 24.68) \% for MHO and $17 \cdot 10$ (95\% CI $14.71,19.78) \%$ for MUHO. The prevalence of the metabolically unhealthy phenotype in males was 16.59 (95\% CI $12 \cdot 23,22 \cdot 11) \%$ among those with normal weight and 40.83 (95\% CI 33.60, 48.47) \% among those with overweight. In females, it was 16.39 (95\% CI 12.59, $21.05) \%$ among those with normal weight and 47.24 (95\% CI 39.62, 54.99)\% among those with overweight.

Tables 1 and 2 show that metabolically unhealthy individuals were older and had higher VAI, SBP, DBP, TAG and us-CRP levels, and lower HDL-C levels, than their metabolically healthy counterparts in both groups (normal weight and overweight) and in both sexes. No significant differences in LTPA and energy intake were found in both sexes, nor for ingestion of macronutrients (data not shown). Body fat percentage was statistically different among the phenotypes in males.

Except for NC in males and BMI in both sexes, all other anthropometric indicators were significantly different among the phenotypes for both sexes, being higher in the metabolically unhealthy groups than in the metabolically healthy groups. Similarly, insulin and HOMA-IR were higher in metabolically unhealthy women than in healthy ones, for both groups (normal weight and overweight).

After the ROC curves were generated, all the anthropometric indicators were able to predict the metabolically unhealthy phenotype, except NC in normal-weight individuals (Table 3). Comparing the AUC for the indicators, VAI showed the highest diagnostic precision in detecting the MUHNW phenotype in both males $(\mathrm{AUC}=0.865)$ and females (AUC $=0.843$ ) and the MUHO phenotype in females (AUC $=0.903$ ). Among males, although the AUC of VAI for the MUHO phenotype was the highest (AUC= 0.830), its accuracy cannot be considered different from WC, WHtR and NC since their 95\% CI overlap. In addition, it can be seen that the AUC values for the indicators WC, WHtR, WHR and NC were similar, regardless of sex and the group evaluated (normal weight and overweight; Table 3).

Associations of VAI and WC with the MUHNW phenotype were found in the adjusted models for males, while for the MUHO phenotype, significant associations were observed for all parameters evaluated, even after adjusting for confounding factors (Table 4).

Among females, we found that VAI, WC and WHtR were associated with the MUHNW phenotype in the adjusted models, while for the MUHO phenotype, we found significant associations with all the anthropometric indicators (Table 5). Tables 4 and 5 show that the strength of the association with the metabolically unhealthy phenotype was higher for VAI than for the other indicators evaluated, especially among individuals with normal weight. In males, the prevalence of the MUHNW phenotype among individuals with VAI above the established cut-off point in the ROC curves (1.487) was 6.74 times the prevalence of individuals with VAI below this value. In females, the prevalence of the MUHNW phenotype among individuals with high VAI (above 1.460) was $7 \cdot 14$ times the prevalence observed among the others.

\section{Discussion}

The results of the current study showed that among the indicators of adiposity evaluated, VAI is the best predictor of the MUHNW phenotype in both sexes and of the MUHO phenotype among women. In the prediction of the MUHO phenotype among men, VAI was diagnostically superior only to WHR. Our findings also revealed that VAI was the indicator most strongly associated with the metabolically unhealthy phenotype, especially among individuals with normal weight.

Evaluation of the diagnostic performance considering the areas below the ROC curves shows that the AUC of VAI for the MUHNW phenotype was 0.865 for males and 0.843 for females, and 0.830 for the MUHO phenotype among men, all classified as good diagnostic performance ${ }^{(29,30)}$. The AUC of VAI for the MUHO phenotype among women was higher than 0.9 , indicating an excellent diagnostic performance ${ }^{(29,30)}$. The performance of the other parameters was classified as only fair or poor.

A study ${ }^{(14)}$ developed to evaluate the feasibility of VAI in identifying individuals with MUHNW in the Chinese population also verified the accuracy of this indicator and its diagnostic superiority in relation to BMI, WC, WHtR and WHR, regardless of the criteria used to define the phenotypes. However, the authors reported AUC for VAI lower than those obtained in our study, ranging from 0.611 to 0.835 depending on the definition criterion.

Kang et $a l{ }^{(9)}$ also evaluated the relationship of VAI with obese phenotypes. Although the main objective of that study was to evaluate the association of VAI with the conversion of the metabolically healthy obese to the metabolically unhealthy obese phenotype, they found that VAI had greater capacity to predict this conversion than WC. The authors suggested that VAI was a more appropriate indicator as a replacement measure in predicting unfavourable metabolic outcomes, possibly due to a more accurate assessment of visceral adiposity.

As already mentioned, our study showed a positive and independent association between VAI and the unhealthy metabolic phenotype in both sexes, and that this 
Table 1 Characteristics of male volunteers according to the different phenotypes. Viçosa, MG, Brazil, 2012-2014

\begin{tabular}{|c|c|c|c|c|c|c|c|c|}
\hline \multirow[b]{2}{*}{ Variable } & \multicolumn{2}{|c|}{ MHNW ( $n$ 186) } & \multicolumn{2}{|c|}{ MUHNW (n 37) } & \multicolumn{2}{|c|}{$\mathrm{MHO}(n 100)$} & \multicolumn{2}{|c|}{ MUHO (n 69) } \\
\hline & Median & IQR & Median & IQR & Median & IQR & Median & IQR \\
\hline Age (years) & $25 \cdot 00^{\mathrm{a}}$ & $22.00-32.00$ & $31.00^{b, c, d}$ & $24.00-49.00$ & $29.00^{C}$ & $23.00-39.50$ & $37.00^{d}$ & $24.00-51.00$ \\
\hline LTPA (min) & 42.50 & $0.00-300.00$ & 0.00 & $0.00-90.00$ & 0.00 & $0.00-250.00$ & 0.00 & $0.00-200.00$ \\
\hline Energy intake $(\mathrm{kJ} / \mathrm{d})$ & 11868.45 & $9952 \cdot 32-14381.07$ & $11210 \cdot 65$ & $8901 \cdot 30-13923.96$ & 11237.08 & $8929 \cdot 61-14421 \cdot 18$ & 11954.32 & $9184.54-14521.79$ \\
\hline BFP (\%) & $15 \cdot 49^{\mathrm{a}}$ & $11.01-19.61$ & $19 \cdot 37^{b}$ & $17.07-22.18$ & $22 \cdot 68^{\mathrm{C}}$ & $19.91-25.78$ & $25 \cdot 01^{d}$ & $22 \cdot 07-29.14$ \\
\hline BMI $\left(\mathrm{kg} / \mathrm{m}^{2}\right)$ & $22 \cdot 61^{a}$ & $21 \cdot 12-23 \cdot 77$ & $23 \cdot 21^{a}$ & $21.64-23.87$ & $26 \cdot 72^{\mathrm{b}, \mathrm{c}}$ & $25.57-28.09$ & $28 \cdot 26^{\mathrm{c}}$ & $26.11-31.33$ \\
\hline VAl & $0.85^{a}$ & $0.61-1.38$ & $2 \cdot 20^{b, d}$ & $1.67-2.85$ & $1 \cdot 19^{c}$ & $0.79-1.54$ & $2 \cdot 51^{d}$ & $1.48-3.56$ \\
\hline WC $(\mathrm{cm})$ & $78.00^{\mathrm{a}}$ & $74.00-81.50$ & $81 \cdot 66^{\mathrm{b}}$ & $78.83-86.00$ & $89.08^{C}$ & $85.75-94.00$ & $95.00^{\mathrm{d}}$ & $89 \cdot 66-102 \cdot 17$ \\
\hline WHtR & $0.44^{\mathrm{a}}$ & $0.42-0.47$ & $0.46^{\mathrm{b}}$ & $0.44-0.48$ & $0.51^{\mathrm{C}}$ & $0.48-0.55$ & $0.55^{\mathrm{d}}$ & $0.51-0.61$ \\
\hline WHR & $0.82^{\mathrm{a}}$ & $0.80-0.86$ & $0.86^{\mathrm{b}}$ & $0.82-0.89$ & $0.88^{\mathrm{C}}$ & $0.83-0.92$ & $0.93^{d}$ & $0.87-0.98$ \\
\hline $\mathrm{NC}(\mathrm{cm})$ & $36 \cdot 83^{a}$ & $35.50-38.00$ & $37.00^{\mathrm{a}}$ & $36.50-38.00$ & $39.00^{\mathrm{b}}$ & $38.00-40.03$ & $40 \cdot 33^{c}$ & $39.00-42.50$ \\
\hline $\mathrm{SBP}(\mathrm{mmHg})$ & $117 \cdot 00^{\mathrm{a}}$ & $111.00-123.00$ & $127 \cdot 75^{\mathrm{b}, \mathrm{d}}$ & $121 \cdot 00-136 \cdot 25$ & $122 \cdot 50^{\mathrm{C}}$ & $115 \cdot 50-129.25$ & $132 \cdot 00^{d}$ & $122.50-137.00$ \\
\hline $\mathrm{DBP}(\mathrm{mmHg})$ & $70 \cdot 50^{\mathrm{a}}$ & $65 \cdot 50-76.50$ & $84.00^{\mathrm{b}, \mathrm{d}}$ & $74.25-89.00$ & $76 \cdot 00^{\mathrm{c}}$ & $71.25-76.00$ & $83.50^{d}$ & $75.50-90.00$ \\
\hline TAG (mg/dl) & $80 \cdot 50^{\mathrm{a}}$ & $62.00-110.00$ & $146 \cdot 00^{b, d}$ & $102.00-173.00$ & $94.00^{\mathrm{C}}$ & $75 \cdot 50-122 \cdot 00$ & $166 \cdot 00^{d}$ & $110 \cdot 00-202.00$ \\
\hline HDL-C (mg/dl) & $47.00^{\mathrm{a}}$ & $40.00-57.00$ & $34.00^{\mathrm{b}, \mathrm{c}}$ & $31.00-39.0$ & $45.00^{\mathrm{a}}$ & $42.00-51.00$ & $36.00^{c}$ & $31.00-40.00$ \\
\hline Glucose (mg/dl) & $81.00^{\mathrm{a}}$ & $77 \cdot 00-86.0$ & $82.00^{\mathrm{a}}$ & $75 \cdot 00-90 \cdot 0$ & $82 \cdot 00^{\mathrm{a}}$ & $78 \cdot 00-86.50$ & $91.00^{\mathrm{b}}$ & $80.00-98.00$ \\
\hline Insulin $(\mu \mathrm{U} / \mathrm{ml})$ & $5.75^{\mathrm{a}}$ & $3.90-8 \cdot 10$ & $6 \cdot 10^{\mathrm{a}, \mathrm{b}}$ & $4.80-7.90$ & $6 \cdot 90^{\mathrm{b}}$ & $4.90-9.80$ & $11.25^{\mathrm{c}}$ & $7 \cdot 30-14 \cdot 20$ \\
\hline HOMA-IR & $1.13^{\mathrm{a}}$ & $0.76-1.65$ & $1.25^{a, b}$ & $1.03-1.53$ & $1.35^{\mathrm{b}}$ & $0.93-2.06$ & $2.53^{\mathrm{C}}$ & $1.54-3.55$ \\
\hline us-CRP (mg/dl) & $0.47^{a}$ & $0.20-1.13$ & $1.34^{\mathrm{b}, \mathrm{c}, \mathrm{d}}$ & $0.57-2.69$ & $0.85^{\mathrm{c}}$ & $0.44-1.89$ & $1 \cdot 31^{d}$ & $0.60-2.63$ \\
\hline
\end{tabular}

MHNW, metabolically healthy normal weight; MUHNW, metabolically unhealthy normal weight; MHO, metabolically healthy overweight; MUHO, metabolically unhealthy overweight; IQR, interquartile range; LTPA, leisuretime physical activity; BFP, body fat percentage; VAl, visceral adiposity index; WC, waist circumference; WHtR, waist-to-height ratio; WHR, waist-to-hip ratio; NC, neck circumference; SBP, systolic blood pressure; DBP, diastolic blood pressure; HDL-C, HDL-cholesterol; HOMA-IR, homeostatic model assessment of insulin resistance; us-CRP, ultra-sensitive C-reactive protein.

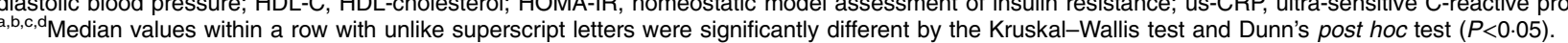


Table 2 Characteristics of female volunteers according to the different phenotypes. Viçosa, MG, Brazil, 2012-2014

\begin{tabular}{|c|c|c|c|c|c|c|c|c|}
\hline \multirow[b]{2}{*}{ Variable } & \multicolumn{2}{|c|}{ MHNW (n 250) } & \multicolumn{2}{|c|}{ MUHNW (n 49) } & \multicolumn{2}{|c|}{$\mathrm{MHO}(n 86)$} & \multicolumn{2}{|c|}{ MUHO ( $n 77)$} \\
\hline & Median & IQR & Median & IQR & Median & IQR & Median & IQR \\
\hline Age (years) & $27 \cdot 50^{a}$ & $23 \cdot 00-38.00$ & $30 \cdot 00^{\mathrm{b}, \mathrm{c}}$ & $24 \cdot 00-48 \cdot 00$ & $33 \cdot 50^{c}$ & $27 \cdot 00-45 \cdot 00$ & $46 \cdot 00^{d}$ & $35 \cdot 00-52 \cdot 00$ \\
\hline LTPA (min) & 0.00 & $0.00-180.00$ & 0.00 & $0.00-180.00$ & 0.00 & $0.00-240.00$ & 0.00 & $0.00-180.00$ \\
\hline Energy intake $(\mathrm{kJ} / \mathrm{d})$ & $9317 \cdot 72$ & $7630 \cdot 04-11338 \cdot 10$ & $9516 \cdot 39$ & $8387 \cdot 12-11478.95$ & 9931.55 & $7643 \cdot 21-12623 \cdot 49$ & $9824 \cdot 70$ & $8425 \cdot 43-11535 \cdot 72$ \\
\hline BFP (\%) & $28 \cdot 61^{a}$ & $25 \cdot 36-31.57$ & $28.97^{a}$ & $25 \cdot 89-32.97$ & $37 \cdot 15^{\mathrm{b}, \mathrm{c}}$ & $34.28-39.10$ & $38.06^{\mathrm{c}}$ & $35 \cdot 31-40.52$ \\
\hline BMI $\left(\mathrm{kg} / \mathrm{m}^{2}\right)$ & $21 \cdot 37^{a}$ & $19.87-23.07$ & $22 \cdot 07^{a}$ & $21.02-24.06$ & $27 \cdot 82^{b, c}$ & $26 \cdot 55-29 \cdot 46$ & $29 \cdot 28^{C}$ & $26.95-31.68$ \\
\hline VAI & $1.08^{\mathrm{a}}$ & $0.77-1.41$ & $2 \cdot 19^{\mathrm{b}}$ & $1.52-3.19$ & $1 \cdot 21^{\mathrm{c}}$ & $0.88-1.64$ & $2.73^{d}$ & $2.06-3.81$ \\
\hline WC (cm) & $71.00^{\mathrm{a}}$ & $67.00-75.00$ & $74.00^{\mathrm{b}}$ & $69.00-81.83$ & $86 \cdot 25^{c}$ & $82.33-90.50$ & $91.66^{d}$ & $86.50-99.50$ \\
\hline WHtR & $0.43^{a}$ & $0.41-0.46$ & $0.46^{\mathrm{b}}$ & $0.43-0.50$ & $0.54^{\mathrm{c}}$ & $0.51-0.58$ & $0.68^{\mathrm{d}}$ & $0.54-0.63$ \\
\hline WHR & $0.74^{a}$ & $0.71-0.78$ & $0.78^{b}$ & $0.73-0.83$ & $0.79^{c}$ & $0.77-0.84$ & $0.88^{d}$ & $0.84-0.92$ \\
\hline $\mathrm{NC}(\mathrm{cm})$ & $31 \cdot 17^{\mathrm{a}}$ & $30 \cdot 50-32 \cdot 17$ & $31.60^{\mathrm{b}}$ & $31.00-32.83 .00$ & $33 \cdot 17^{\mathrm{C}}$ & $32 \cdot 16-34 \cdot 67$ & $34.83^{\mathrm{d}}$ & $33.83-36.50$ \\
\hline $\mathrm{SBP}(\mathrm{mmHg})$ & $105 \cdot 50^{\mathrm{a}}$ & $100 \cdot 00-113.50$ & $114.50^{\mathrm{b}, \mathrm{c}}$ & $105.50-124.00$ & $113 \cdot 00^{c}$ & $107 \cdot 00-121.50$ & $123 \cdot 25^{d}$ & $115 \cdot 25-132 \cdot 00$ \\
\hline DBP $(\mathrm{mmHg})$ & $69 \cdot 00^{a}$ & $64.50-74.50$ & $74.00^{\mathrm{b}, \mathrm{c}}$ & $68.00-85.00$ & $74.50^{c}$ & $70.00-78.50$ & $82.50^{\mathrm{d}}$ & $76.25-88.75$ \\
\hline TAG $(\mathrm{mg} / \mathrm{dl})$ & $80 \cdot 00^{a}$ & $64.00-108.00$ & $152 \cdot 00^{\mathrm{b}, \mathrm{c}}$ & $100 \cdot 00-186 \cdot 00$ & $84.50^{a}$ & $64.00-109 \cdot 00$ & $154.00^{c}$ & $104 \cdot 00-210 \cdot 00$ \\
\hline HDL-C (mg/dl) & $58.00^{a}$ & $50.00-71.00$ & $47 \cdot 00^{\mathrm{b}}$ & $39.00-54.00$ & $56 \cdot 50^{a}$ & $49.00-66.00$ & $42 \cdot 00^{\mathrm{C}}$ & $38.00-48.00$ \\
\hline Glucose (mg/dl) & $80 \cdot 00^{a}$ & $76.00-85.00$ & $83.00^{a}$ & $76.00-90.00$ & $82 \cdot 00^{a}$ & $76.00-87.00$ & $89 \cdot 00^{\mathrm{b}}$ & $81 \cdot 00-100 \cdot 00$ \\
\hline Insulin $(\mu \mathrm{U} / \mathrm{ml})$ & $6 \cdot 70^{\mathrm{a}}$ & $4.60-9.40$ & $8 \cdot 20^{\mathrm{b}, \mathrm{c}}$ & $5 \cdot 80-13 \cdot 30$ & $7.90^{\mathrm{c}}$ & $5 \cdot 60-10.50$ & $11 \cdot 25^{\mathrm{d}}$ & $8.00-14.90$ \\
\hline HOMA-IR & $1 \cdot 30^{\mathrm{a}}$ & $0.90-1.95$ & $1.79^{\mathrm{b}, \mathrm{c}}$ & $1.25-2.67$ & $1.55^{c}$ & $1 \cdot 13-2 \cdot 19$ & $2.62^{d}$ & $1.75-3.83$ \\
\hline us-CRP (mg/dl) & $0.97^{a}$ & $0.37-2.23$ & $2 \cdot 69^{b, c}$ & $1.28-5.2$ & $1 \cdot 27^{a}$ & $0.49-2.50$ & $2 \cdot 36^{c}$ & $1.21-4.09$ \\
\hline
\end{tabular}

MHNW, metabolically healthy normal weight; MUHNW, metabolically unhealthy normal weight; MHO, metabolically healthy overweight; MUHO, metabolically unhealthy overweight; IQR, interquartile range; LTPA, leisure-time physical activity; BFP, body fat percentage; VAI, visceral adiposity index; WC, waist circumference; WHtR, waist-to-height ratio; WHR, waist-to-hip ratio; NC, neck circumference; SBP, systolic blood pressure; DBP, diastolic blood pressure; HDL-C, HDL-cholesterol; HOMA-IR, homeostatic model assessment of insulin resistance; us-CRP, ultra-sensitive C-reactive protein.

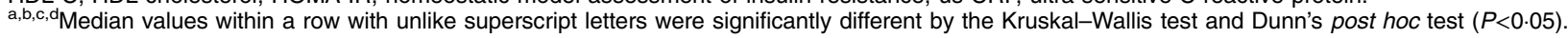

association was stronger among individuals with normal weight. Although this finding needs to be confirmed by other studies, it demonstrates the potential of VAI to identify individuals with normal weight at cardiometabolic risk.

The association between VAI and the metabolically unhealthy phenotype, both among normal-weight individuals and those with excess weight, confirms that increased visceral adiposity is related to the occurrence of an unfavourable metabolic profile. Similarly, Du et $a l{ }^{(14)}$ found that the individuals with the highest VAI had the poorest metabolic profile. These results highlight the applicability of VAI as an important marker of cardiometabolic risk.

We should keep aware of metabolic abnormalities that may or may not be associated with obesity because there is evidence that normal and metabolically unhealthy individuals are at increased risk for CVD and type 2 diabetes $^{(12,31,32)}$. Because individuals of this group are not overweight, most of the time they are not the target of screening programmes and, therefore, they fail to receive the appropriate interventions.

The associations found between the other indicators of adiposity evaluated and the metabolically unhealthy phenotype allow us to infer that they can also identify central obesity, even though with less precision than VAI. This difference may occur because these indicators are based only on anthropometric measures, while VAI includes anthropometric and lipid parameters in its formulation, leading to better detection of tissue dysfunction. On this point, it is important to point out that the two lipid parameters used in VAI formulation (i.e. TAG and HDL-C) are also used in the determination of metabolic phenotypes. As result, higher correlations of metabolically unhealthy phenotypes with VAI than with the other indicators evaluated were expected. However, we would like to emphasize that our main objective was to compare VAI predictive capacity by nutritional status and sex. Interestingly, we identified that VAI is more strongly associated with the metabolically unhealthy phenotype among normal-weight than overweight individuals, showing that this index can be very useful in the MUHNW phenotype identification.

The prevalence of MUHNW phenotype was approximately $16 \%$, with values very close between men and women. These prevalences are similar to those described by Aung et $a l .^{(12)}$ and Ryoo et al. ${ }^{(33,34)}$, which were $12 \cdot 8$, $21 \cdot 1$ and $13.30 \%$, respectively. Although these authors did not stratify the prevalence by sex, the criteria used to define the phenotype were similar to those of our study ${ }^{(7)}$, allowing comparisons to be made. We found MUHO prevalences of 40.83 and $47.24 \%$ for males and females, respectively, higher than that reported by Wu et al. ${ }^{(32)}$, which was $34 \cdot 1 \%$.

Metabolically unhealthy individuals were older, as observed in other studies ${ }^{(3,10,14,35)}$. This finding suggests that age may influence the metabolic profile, and due attention should be given to individuals as they age.

Our study has some limitations. First, the sample size may have reduced its power to detect associations, especially by having stratified the analyses according to sex and nutritional status. Another aspect is that the crosssectional nature of the study limits the interpretation in relation to the directionality of the associations found. 
Table 3 Areas under the receiver-operating characteristic curves and cut-off points of the visceral adiposity index and anthropometric indicators to predict metabolically unhealthy phenotype among normal-weight and overweight individuals, according to sex. Viçosa, MG, Brazil, 2012-2014

\begin{tabular}{|c|c|c|c|c|c|c|c|c|c|c|}
\hline & \multicolumn{5}{|c|}{ MUHNW } & \multicolumn{5}{|c|}{ MUHO } \\
\hline & AUC & $95 \% \mathrm{Cl}$ & Cut-off point & Sens. (\%) & Spec. (\%) & AUC & $95 \% \mathrm{Cl}$ & Cut-off point & Sens. (\%) & Spec. (\%) \\
\hline \multicolumn{11}{|l|}{ Males } \\
\hline VAl & 0.865 & $0.803,0.927$ & 1.487 & 78.38 & 77.96 & 0.830 & $0.762,0.897$ & 1.483 & $75 \cdot 36$ & 74.00 \\
\hline$W C(\mathrm{~cm})$ & 0.690 & $0.602,0.778$ & 80.000 & 64.86 & 64.52 & 0.684 & $0.599,0.769$ & 91.500 & $65 \cdot 22$ & 64.00 \\
\hline WHtR & 0.683 & $0.595,0.771$ & 0.454 & $62 \cdot 16$ & 61.29 & 0.682 & $0.598,0.766$ & 0.529 & 66.67 & 66.00 \\
\hline WHR & 0.662 & $0.564,0.760$ & 0.843 & 64.86 & 64.67 & 0.649 & $0.563,0.735$ & 0.897 & $62 \cdot 32$ & 62.00 \\
\hline $\mathrm{NC}(\mathrm{cm})$ & 0.579 & $0.486,0.673$ & 37.000 & $62 \cdot 16$ & 51.08 & 0.696 & $0.614,0.777$ & 39.660 & $65 \cdot 22$ & 63.00 \\
\hline \multicolumn{11}{|l|}{ Females } \\
\hline VAl & 0.843 & $0.778,0.908$ & 1.460 & 77.55 & 77.60 & 0.903 & $0.858,0.948$ & 1.835 & 80.52 & 80.23 \\
\hline$W C(\mathrm{~cm})$ & 0.638 & $0.546,0.730$ & $72 \cdot 760$ & $61 \cdot 22$ & $61 \cdot 20$ & 0.697 & $0.616,0.779$ & 89.000 & 64.94 & $62 \cdot 79$ \\
\hline $\mathrm{WH} \mathrm{tR}$ & 0.655 & $0.567,0.742$ & 0.447 & 61.22 & 61.20 & 0.703 & $0.622,0.783$ & 0.559 & 66.23 & $65 \cdot 12$ \\
\hline WHR & 0.644 & $0.554,0.734$ & 0.755 & 61.22 & 58.63 & 0.753 & $0.677,0.830$ & 0.839 & 72.73 & 71.76 \\
\hline $\mathrm{NC}(\mathrm{cm})$ & 0.591 & $0.504,0.678$ & 31.500 & 59.18 & 54.03 & 0.711 & $0.631,0.791$ & 34.000 & $73 \cdot 68$ & $60 \cdot 00$ \\
\hline
\end{tabular}

MUHNW, metabolically unhealthy normal weight; MUHO, metabolically unhealthy overweight; AUC, area under the ROC (receiver-operating characteristic) curve; Sens., sensitivity; Spec., specificity; VAI, visceral adiposity index; WC, waist circumference; WHtR, waist-to-height ratio; WHR, waist-to-hip ratio; NC, neck circumference. 
Table 4 Poisson regression analysis for associations of the visceral adiposity index and anthropometric indicators with metabolically unhealthy phenotype among normal-weight and overweight male volunteers. Viçosa, MG, Brazil, 2012-2014

\begin{tabular}{|c|c|c|c|c|c|c|}
\hline & \multicolumn{3}{|c|}{ Crude } & \multicolumn{3}{|c|}{ Adjusted* } \\
\hline & PR & $95 \% \mathrm{Cl}$ & $P$ & PR & $95 \% \mathrm{Cl}$ & $P$ \\
\hline \multicolumn{7}{|l|}{ MUHNW } \\
\hline VAI & 7.92 & $3.81,16.46$ & $<0.001$ & $6 \cdot 74$ & $3.15,14.42$ & $<0.001$ \\
\hline $\mathrm{WC}(\mathrm{cm})$ & 2.73 & $1.39,5.36$ & 0.004 & $2 \cdot 14$ & $1.07,4.25$ & 0.030 \\
\hline WHtR & $2 \cdot 17$ & $1 \cdot 18,4.00$ & 0.013 & 1.40 & $0.74,2.7$ & 0.303 \\
\hline WHR & $2 \cdot 68$ & $1.44,4.98$ & 0.002 & 1.83 & $0.97,3.46$ & 0.063 \\
\hline $\mathrm{NC}(\mathrm{cm})$ & 1.57 & $0.85,2.90$ & 0.148 & 1.70 & $0.96,3.02$ & 0.070 \\
\hline \multicolumn{7}{|l|}{ MUHO } \\
\hline VAl & 3.57 & $2 \cdot 26,5 \cdot 64$ & $<0.001$ & 3.33 & $2 \cdot 10,5 \cdot 29$ & $<0.001$ \\
\hline WC (cm) & 2.04 & $1.37,3.02$ & $<0.001$ & 1.88 & $1 \cdot 20,2 \cdot 92$ & 0.005 \\
\hline $\mathrm{WHtR}$ & $2 \cdot 22$ & $1.49,3.32$ & $<0.001$ & $2 \cdot 10$ & $1.34,3.29$ & 0.001 \\
\hline WHR & 1.80 & $1.22,2.64$ & 0.003 & 1.68 & $1.05,2.68$ & 0.031 \\
\hline $\mathrm{NC}(\mathrm{cm})$ & 1.88 & $1.28,2.76$ & 0.001 & $1 \cdot 761$ & $1 \cdot 19,2.60$ & 0.004 \\
\hline
\end{tabular}

PR, prevalence ratio; MUHNW, metabolically unhealthy normal weight; VAl, visceral adiposity index; WC, waist circumference; WHtR, waist-to-height ratio; WHR, waist-to-hip ratio; NC, neck circumference; MUHO, metabolically unhealthy overweight.

${ }^{*}$ Adjusted for age, schooling and socio-economic status.

Table 5 Poisson regression analysis for associations of the visceral adiposity index and anthropometric indicators with metabolically unhealthy phenotype among normal-weight and overweight female volunteers. Viçosa, MG, Brazil, 2012-2014

\begin{tabular}{|c|c|c|c|c|c|c|}
\hline & \multicolumn{3}{|c|}{ Crude } & \multicolumn{3}{|c|}{ Adjusted $^{*}$} \\
\hline & PR & $95 \% \mathrm{Cl}$ & $P$ & PR & $95 \% \mathrm{Cl}$ & $P$ \\
\hline \multicolumn{7}{|l|}{ MUHNW } \\
\hline VAI & 7.53 & $4.03,14.09$ & $<0.001$ & $7 \cdot 14$ & $3.79,13.44$ & $<0.001$ \\
\hline WC (cm) & $2 \cdot 14$ & $1 \cdot 26,3 \cdot 62$ & 0.005 & 1.84 & $1.03,3.30$ & 0.039 \\
\hline $\mathrm{WHtR}$ & $2 \cdot 13$ & $1.26,3.62$ & 0.005 & 1.85 & $1.04,3.28$ & 0.036 \\
\hline WHR & 1.94 & $1 \cdot 15,3 \cdot 30$ & 0.014 & 1.64 & $0.95,2.82$ & 0.076 \\
\hline $\mathrm{NC}(\mathrm{cm})$ & 1.54 & $0.91,2.60$ & 0.106 & 1.31 & $0.77,2.21$ & 0.318 \\
\hline \multicolumn{7}{|l|}{ MUHO } \\
\hline VAI & 4.39 & $2 \cdot 73,7.06$ & $<0.001$ & 3.80 & $2 \cdot 36,6 \cdot 12$ & $<0.001$ \\
\hline $\mathrm{WC}(\mathrm{cm})$ & 1.83 & $1 \cdot 28,2 \cdot 61$ & 0.001 & 1.55 & $1 \cdot 11,2 \cdot 18$ & 0.011 \\
\hline WHtR & 1.99 & $1 \cdot 38,2 \cdot 85$ & $<0.001$ & 1.49 & $1 \cdot 03,2 \cdot 15$ & 0.034 \\
\hline WHR & 2.63 & $1.77,3.92$ & $<0.001$ & 1.98 & $1.28,3.08$ & 0.002 \\
\hline $\mathrm{NC}(\mathrm{cm})$ & $2 \cdot 20$ & $1.47,3.30$ & $<0.001$ & 1.91 & $1 \cdot 28,2 \cdot 81$ & 0.001 \\
\hline
\end{tabular}

PR, prevalence ratio; MUHNW, metabolically unhealthy normal weight; VAI, visceral adiposity index; WC, waist circumference; WHtR, waist-to-height ratio; WHR, waist-to-hip ratio; NC, neck circumference; MUHO, metabolically unhealthy overweight.

${ }^{*}$ Adjusted for age, schooling and socio-economic status.

Despite these limitations, the present study has many strengths, including the more comprehensive criteria used to define the phenotypes which consider not only components of the metabolic syndrome, thereby detecting a greater range of metabolic abnormalities. The performance of stratified analyses by sex is another strength of the study, since it has been shown that men and women differ in the accumulation of visceral fat. Finally, the methodological rigour of data collection, which included trained interviewers, standardized protocols and anthropometric measurements taken by a single examiner, ensured the validity of the results and their potential for application.

\section{Conclusion}

In conclusion, the present study results showed that, in general, VAI has a better capacity of predicting the metabolically unhealthy phenotype than conventional anthropometric indicators in both sexes. We also found that VAI is most strongly associated with metabolically unhealthy phenotype among normal-weight individuals. The main implication of these findings is that, by using an index calculated from simple measures, it is possible to predict accurately the presence of an unfavourable cardiometabolic profile. Particularly, the identification of individuals with MUHNW phenotype allows early 
interventions to prevent future occurrence of CVD and diabetes.

The evidence from the present study therefore suggests that efforts be directed toward the identification of individuals with normal weight and at cardiometabolic risk, because although they have a high potential for the development of diabetes and CVD, they are generally disregarded in screening and prevention programmes.

\section{Acknowledgements}

Acknowledgements: The authors thank all the volunteers who contributed to the study, as well as the National Council for Scientific and Technological Development (CNPq) and the Foundation for Supporting Research in the State of Minas Gerais (FAPEMIG) for funding the project. Financial support: This work was supported by CNPq (grant number 481418/2011-3) and FAPEMIG (grant number APQ-00296-12). The funding agencies had no role in the design, analysis or writing of this article. Conflict of interest: The authors declare no conflict of interest. Authorship: F.G.F. contributed to the data collection and analyses, wrote the initial draft of the manuscript and assembled the final version. L.L.J. and G.Z.L. contributed to the study design, analysis design and data analyses, and participated in the approval of the final version of the manuscript. D.C.G.S. contributed to the data collection and analyses and participated in the approval of the final version of the manuscript. Etbics of human subject participation: This study was conducted according to the guidelines laid down in the Declaration of Helsinki and all procedures involving human subjects were approved by the Research Ethics Committee of the Federal University of Viçosa (Official Letter 02/2013). Written informed consent was obtained from all subjects.

\section{References}

1. Malik VS, Willett WC \& Hu FB (2013) Global obesity: trends, risk factors and policy implications. Nat Rev Endocrinol 20, $13-27$.

2. Nguyen T \& Lau DCW (2012) The obesity epidemic and its impact on hypertension. Can J Cardiol 28, 326-333.

3. Mirzaei B, Abdi H, Serahati S et al. (2017) Cardiovascular risk in different obesity phenotypes over a decade followup: Tehran Lipid and Glucose Study. Atherosclerosis 258 , 65-71.

4. Karelis AD, St-Pierre DH, Conus F et al. (2004) Metabolic and body composition factors in subgroups of obesity: what do we know? J Clin Endocrinol Metab 89, 2569-2575.

5. Global Burden of Metabolic Risk Factors for Chronic Diseases Collaboration (BMI Mediated Effects), Lu Y, Hajifathalian K et al. (2014) Metabolic mediators of the effects of body-mass index, overweight, and obesity on coronary heart disease and stroke: a pooled analysis of 97 prospective cohorts with 1.8 million participants. Lancet $\mathbf{3 8 3}$, 970-983.
6. Ruderman N, Chisholm D \& Pi-Sunyer X (1998) The metabolically obese, normal-weight individual revisited. Diabetes 47, 699-713.

7. Wildman R, Muntner P, Reynolds K et al. (2008) The obese without cardiometabolic risk factor clustering and the normal weight with cardiometabolic risk factor clustering: prevalence and correlates of 2 phenotypes among the US population (NHANES 1999-2004). Arch Intern Med 168, $1617-1624$.

8. Hwang Y-C, Hayashi T, Fujimoto WY et al. (2015) Visceral abdominal fat accumulation predicts the conversion of metabolically healthy obese subjects to an unhealthy phenotype. Int J Obes (Lond) 39, 1365-1370.

9. Kang YM, Jung CH, Cho YK et al. (2015) Visceral adiposity index predicts the conversion of metabolically healthy obesity to an unhealthy phenotype. PLoS One 12, e0179635.

10. Kim NH, Seo JA, Cho H et al. (2016) Risk of the development of diabetes and cardiovascular disease in metabolically healthy obese people: the Korean Genome and Epidemiology Study. Medicine (Baltimore) 95, e3384.

11. Kabat GC, Wu WY-Y, Bea JW et al. (2017) Metabolic phenotypes of obesity: frequency, correlates and change over time in a cohort of postmenopausal women. Int $J$ Obes (Lond) 41, 170-177.

12. Aung K, Lorenzo C, Hinojosa MA et al. (2014) Risk of developing diabetes and cardiovascular disease in metabolically unhealthy normal-weight and metabolically healthy obese individuals. J Clin Endocrinol Metab 99, 462-468.

13. Phillips CM, Dillon C, Harrington JM et al. (2013) Defining metabolically healthy obesity: role of dietary and lifestyle factors. PLoS One 8, e76188.

14. Du T, Yu X, Zhang J et al. (2015) Lipid accumulation product and visceral adiposity index are effective markers for identifying the metabolically obese normal-weight phenotype. Acta Diabetol 52, 855-863.

15. Samocha-Bonet D, Dixit VD, Kahn CR et al. (2014) Metabolically healthy and unhealthy obese - the 2013 Stock Conference report. Obes Rev 15, 697-708.

16. Amato MC, Giordano C, Galia M et al. (2010) Visceral adiposity index: a reliable indicator of visceral fat function associated with cardiometabolic risk. Diabetes Care 33, 920-922.

17. Segheto W, Silva DCG, Coelho FA et al. (2015) Body adiposity index and associated factors in adults: method and logistics of a population-based study. Nutr Hosp 32, 101-109.

18. Barbosa MB, Pereira CV, da Cruz DT et al. (2018) Prevalence and factors associated with alcohol and tobacco use among non-institutionalized elderly persons. Rev Bras Geriatr Gerontol 21, 125-135.

19. Amato MC \& Giordano C (2013) Clinical indications and proper use of visceral adiposity index. Nutr Metab Cardiovasc Dis 23, e31-e32.

20. Matthews DR, Hosker JP, Rudenski AS et al. (1985) Homeostasis model assessment: insulin resistance and $\beta$-cell function from fasting plasma glucose and insulin concentrations in man. Diabetologia 28, 412-419.

21. World Health Organization (2000) Obesity: Preventing and Managing the Global Epidemic. Report of a WHO Consultation. WHO Technical Report Series no. 894. Geneva: WHO.

22. Ashwell M \& Hsieh SD (2005) Six reasons why the waist-toheight ratio is a rapid and effective global indicator for health risks of obesity and how its use could simplify the international public health message on obesity. Int J Food Sci Nutr 56, 303-307.

23. Ben-noun L, Sohar E \& Laor A (2001) Neck circumference as a simple screening measure for identifying overweight and obese patients. Obes Res 9, 470-477. 
24. Jackson AS, Pollock ML \& Ward A (1980) Generalized equations for predicting body density of women. Med Sci Sports Exerc 12, 175-181.

25. Jackson AS \& Pollock ML (1978) Generalized equations for predicting body density of men. Br J Nutr $\mathbf{4 0}$, 497-504.

26. Siri WE (1956) The gross composition of the body. Adv Biol Med Phys 4, 239-280

27. Haskell WL, Lee I-M, Pate RR et al. (2007) Physical activity and public health updated recommendation for adults from the American College of Sports Medicine and the American Heart Association. Med Sci Sport Exerc 39, 1423-1434.

28. da Silva DCG, Segheto W, de Lima MFC et al. (2018) Using the method of triads in the validation of a food frequency questionnaire to assess the consumption of fatty acids in adults. J Hum Nutr Diet 31, 85-95.

29. Janghorbani M, Aminorroaya A \& Amini M (2017) Comparison of different obesity indices for predicting incident hypertension. High Blood Press Cardiovasc Prev 24, 157-166.

30. Janghorbani M \& Amini M (2016) The visceral adiposity index in comparison with easily measurable anthropometric markers did not improve prediction of diabetes. Can $J$ Diabetes 40, 393-398.

31. Janghorbani M, Salamat MR, Amini M et al. (2017) Risk of diabetes according to the metabolic health status and degree of obesity. Diabetes Metab Syndr Clin Res Rev S1871, 4021-4022.

32. Wu S, Fisher-Hoch SP, Reninger B et al. (2016) Metabolic health has greater impact on diabetes than simple overweight/obesity in Mexican Americans. J Diabetes Res 2016, 4094876.

33. Ryoo J-H, Park SK, Ye S et al. (2015) Estimation of risk for diabetes according to the metabolically healthy status stratified by degree of obesity in Korean men. Endocrine 50, 650-658.

34. Ryoo J-H, Park SK, Oh C-M et al. (2017) Evaluating the risk of hypertension according to the metabolic health status stratified by degree of obesity. J Am Soc Hypertens 11, 20-27.

35. Pimentel A de C, Scorsatto M, Moraes de Oliveira GM et al. (2015) Characterization of metabolically healthy obese Brazilians and cardiovascular risk prediction. Nutrition 31, 827-833. 